\title{
Halpern-type proximal point algorithm in complete CAT(0) metric spaces
}

\author{
Mohammad Taghi Heydari and Sajad Ranjbar
}

\begin{abstract}
First, Halpern-type proximal point algorithm is introduced in complete CAT(0) metric spaces. Then, Browder convergence theorem is considered for this algorithm and also we prove that Halpern-type proximal point algorithm converges strongly to a zero of the operator.
\end{abstract}

\section{Introduction}

One of the most important parts in nonlinear and convex analysis is monotone operator theory. It has an essential role in convex analysis, optimization, variational inequalities, semigroup theory and evolution equations. A zero of a maximal monotone operator is a solution of variational inequality associated to the monotone operator also an equilibrium point of an evolution equation governed by the monotone operator as well as a solution of a minimization problem for a convex function when the monotone operator is a subdifferential of the convex function. Therefore existence and approximation of a zero of a maximal monotone operator is the center of consideration of many recent researchers. The most popular method for approximation of a zero of a maximal monotone operator is the proximal point algorithm which was introduced by Martinet [30] and Rockafellar [32]. Rockafellar [32] showed the weak convergence of the sequence generated by the proximal point algorithm to a zero

Key Words: Hadamard space, Maximal monotone operator, Halpern-type proximal point algorithm, w-convergence, Strong convergence.

2010 Mathematics Subject Classification: Primary 47H05, 47J05; Secondary 47J20, $65 \mathrm{~K} 05$.

Received:10.10.2015

Accepted:15.12.2015 
of the maximal monotone operator in Hilbert spaces. Güler's counterexample [21] showed that the sequence generated by the proximal point algorithm does not necessarily converge strongly even if the maximal monotone operator is the subdifferential of a convex, proper, and lower semicontinuous function. Xu [35] and Kamimura and Takahashi [23] introduced a Halpern-type proximal point algorithm, which guarantees the strong convergence in Hilbert space. For some generalization in Hilbert spaces the reader can consult $[6,12,17,24,25,33]$. Proximal point algorithm introduced by Bačák [3] for the case of convex functions in Hadamard spaces. In the general cases, this algorithm is organized in Hadamard spaces by Khatibzadeh and Ranjbar [26] for the monotone operators (also, see [31]). In this paper by using of the duality theory introduced in [2], we consider maximal monotone operators and Halpern-type proximal point algorithm on Hadamard spaces and prove the strong convergence of Halperntype proximal point algorithm in this nonlinear version of Hilbert spaces (i.e. complete $\mathrm{CAT}(0)$ spaces).

\section{Preliminaries}

Let $(X, d)$ be a metric space and $x, y \in X$. A geodesic path joining $x$ to $y$ is an isometry $c:[0, d(x, y)] \longrightarrow X$ such that $c(0)=x, c(d(x, y))=y$. The image of a geodesic path joining $x$ to $y$ is called a geodesic segment between $x$ and $y$. The metric space $(X, d)$ is said to be a geodesic space if every two points of $X$ are joined by a geodesic, and $X$ is said to be an uniquely geodesic space if there is exactly one geodesic joining $x$ and $y$ for each $x, y \in X$. ity:

A geodesic space $(X, d)$ is a $\operatorname{CAT}(0)$ space if satisfies the following inequal-

$C N$-inequality: If $x, y_{0}, y_{1}, y_{2} \in X$ such that $d\left(y_{0}, y_{1}\right)=d\left(y_{0}, y_{2}\right)=\frac{1}{2} d\left(y_{1}, y_{2}\right)$, then

$$
d^{2}\left(x, y_{0}\right) \leq \frac{1}{2} d^{2}\left(x, y_{1}\right)+\frac{1}{2} d^{2}\left(x, y_{2}\right)-\frac{1}{4} d^{2}\left(y_{1}, y_{2}\right)
$$

A complete CAT(0) space is called a Hadamard space. It is known that $\mathrm{CAT}(0)$ spaces are uniquely geodesic spaces. For other equivalent definitions and basic properties, we refer the reader to the standard texts such as $[10$, 14, 20, 22]. Some examples of CAT(0) spaces are pre-Hilbert spaces (see [10]), $\mathbb{R}$-trees (see [27]), Euclidean buildings (see [13]), the complex Hilbert ball with a hyperbolic metric (see [19]), Hadamard manifolds and many others.

For all $x$ and $y$ belong to a $\operatorname{CAT}(0)$ space $X$, we write $(1-t) x \oplus$ ty for the unique point $z$ in the geodesic segment joining from $x$ to $y$ such that $d(z, x)=t d(x, y)$ and $d(z, y)=(1-t) d(x, y)$. Set $[x, y]=\{(1-t) x \oplus t y: t \in$ $[0,1]\}$, a subset $C$ of $X$ is called convex if $[x, y] \subseteq C$ for all $x, y \in C$.

The following technical lemma is well-known in $\mathrm{CAT}(0)$ spaces. 
Lemma 2.1. [16] Let $(X, d)$ be a $C A T(0)$ space. Then, for all $x, y, z \in X$ and all $t \in[0,1]$ :

(1) $d^{2}(t x \oplus(1-t) y, z) \leq t d^{2}(x, z)+(1-t) d^{2}(y, z)-t(1-t) d^{2}(x, y)$,

(2) $\quad d(t x \oplus(1-t) y, z) \leq t d(x, z)+(1-t) d(y, z)$,

In addition, by using (1), we have

$$
d(t x \oplus(1-t) y, t x \oplus(1-t) z) \leq(1-t) d(y, z) .
$$

A concept of convergence in complete $\mathrm{CAT}(0)$ spaces was introduced by Lim [29] that is called $\Delta$-convergence as follows:

Let $\left(x_{n}\right)$ be a bounded sequence in complete $\operatorname{CAT}(0)$ space $(X, d)$ and $x \in X$. Set $r\left(x,\left(x_{n}\right)\right):=\lim \sup _{n \rightarrow \infty} d\left(x, x_{n}\right)$. The asymptotic radius of $\left(x_{n}\right)$ is given by $r\left(\left(x_{n}\right)\right):=\inf \left\{r\left(x,\left(x_{n}\right)\right): x \in X\right\}$ and the asymptotic center of $\left(x_{n}\right)$ is the set $A\left(\left(x_{n}\right)\right):=\left\{x \in X: r\left(x,\left(x_{n}\right)\right)=r\left(\left(x_{n}\right)\right)\right\}$. It is known that in the complete CAT(0) spaces, $A\left(\left(x_{n}\right)\right)$ consists of exactly one point (see [28]). A sequence $\left(x_{n}\right)$ in the complete $\operatorname{CAT}(0)$ space $(X, d)$ is said $\Delta$-convergent to $x \in X$ if $A\left(\left(x_{n_{k}}\right)\right)=\{x\}$ for every subsequence $\left(x_{n_{k}}\right)$ of $\left(x_{n}\right)$. The concept of $\Delta$-convergence has been studied by many authors (e.g. [16, 18]).

Berg and Nikolaev [4] have introduced the concept of quasilinearization for $\operatorname{CAT}(0)$ space $X$. They denote a pair $(a, b) \in X \times X$ by $\overrightarrow{a b}$ and called it a vector. Then the quasilinearization map $\langle\rangle:.(X \times X) \times(X \times X) \rightarrow \mathbb{R}$ is defined by

$$
\langle\overrightarrow{a b}, \overrightarrow{c d}\rangle=\frac{1}{2}\left(d^{2}(a, d)+d^{2}(b, c)-d^{2}(a, c)-d^{2}(b, d)\right), \quad(a, b, c, d \in X) .
$$

It can be easily verified that $\langle a b, a b\rangle=d^{2}(a, b),\langle b a, c d\rangle=-\langle a b, c d\rangle$ and $\langle a b, c d\rangle=\langle a e, c d\rangle+\langle e b, c d\rangle$ are satisfied for all $a, b, c, d, e \in X$. Also, we can formally add compatible vectors, more precisely $\overrightarrow{a c}+\overrightarrow{c b}=\overrightarrow{a b}$, for all $a, b, c \in X$. We say that $X$ satisfies the Cauchy-Schwarz inequality if

$$
\langle\overrightarrow{a b}, \overrightarrow{c d}\rangle \leq d(a, b) d(c, d), \quad(a, b, c, d \in X) .
$$

It is known ([4], Corollary 3$)$ that a geodesically connected metric space is a CAT(0) space if and only if it satisfies the Cauchy-Schwarz inequality.

Ahmadi Kakavandi and Amini [2] have introduced the concept of dual space of a complete $\operatorname{CAT}(0)$ space $X$, based on a work of Berg and Nikolaev [4], as follows.

Consider the map $\Theta: \mathbb{R} \times X \times X \rightarrow C(X, \mathbb{R})$ defined by

$$
\Theta(t, a, b)(x)=t\langle\overrightarrow{a b}, \overrightarrow{a x}\rangle, \quad(t \in \mathbb{R}, a, b, x \in X),
$$

where $C(X, \mathbb{R})$ is the space of all continuous real-valued functions on $X$. Then the Cauchy-Schwarz inequality implies that $\Theta(t, a, b)$ is a Lipschitz function 
with Lipschitz semi-norm $L(\Theta(t, a, b))=|t| d(a, b) \quad(t \in \mathbb{R}, a, b \in X)$, where $L(\varphi)=\sup \left\{\frac{\varphi(x)-\varphi(y)}{d(x, y)}: x, y \in X, x \neq y\right\}$ is the Lipschitz semi-norm for any function $\varphi: X \rightarrow \mathbb{R}$. A pseudometric $D$ on $\mathbb{R} \times X \times X$ is defined by

$$
D((t, a, b),(s, c, d))=L(\Theta(t, a, b)-\Theta(s, c, d)), \quad(t, s \in \mathbb{R}, a, b, c, d \in X) .
$$

For a Hadamard space $(X, d)$, the pseudometric space $(\mathbb{R} \times X \times X, D)$ can be considered as a subspace of the pseudometric space of all real-valued Lipschitz functions $(\operatorname{Lip}(X, \mathbb{R}), L)$. It is obtained that $D((t, a, b),(s, c, d))=0$ if and only if $t\langle\overrightarrow{a b}, \overrightarrow{x y}\rangle=s\langle\overrightarrow{c d}, \overrightarrow{x y}\rangle$, for all $x, y \in X[2$, Lemma 2.1]. Then, $D$ can impose an equivalent relation on $\mathbb{R} \times X \times X$, where the equivalence class of $(t, a, b)$ is

$$
[t \overrightarrow{a b}]=\{s \overrightarrow{c d}: D((t, a, b),(s, c, d))=0\} .
$$

The set $X^{*}=\{[\overrightarrow{a \vec{b}}]:(t, a, b) \in \mathbb{R} \times X \times X\}$ is a metric space with metric $D([t \overrightarrow{a b}],[s \overrightarrow{c c}]):=D((t, a, b),(s, c, d))$, which is called the dual space of $(X, d)$. It is clear that $[\overrightarrow{a \vec{a}}]=[\overrightarrow{b b}]$ for all $a, b \in X$. Fix $o \in X$, we write $\mathbf{0}=[\overrightarrow{o b}]$ as the zero of the dual space. In [2], it is shown that the dual of a closed and convex subset of Hilbert space $H$ with nonempty interior is $H$ and $t(b-a) \equiv[t \overrightarrow{a b}]$ for all $t \in \mathbb{R}, a, b \in H$. Note that $X^{*}$ acts on $X \times X$ by

$$
\left\langle x^{*}, \overrightarrow{x y}\right\rangle=t\langle\overrightarrow{a b}, \overrightarrow{x y}\rangle, \quad\left(x^{*}=[t \overrightarrow{a b}] \in X, x, y \in X\right) .
$$

Also, we use the following notation:

$$
\left\langle\alpha x^{*}+\beta y^{*}, \overrightarrow{x y}\right\rangle:=\alpha\left\langle x^{*}, \overrightarrow{x y}\right\rangle+\beta\left\langle y^{*}, \overrightarrow{x y}\right\rangle, \quad\left(\alpha, \beta \in \mathbb{R}, x, y \in X, x^{*}, y^{*} \in X^{*}\right) .
$$

Introducing of a dual for a $\mathrm{CAT}(0)$ space implies a concept of weak convergence with respect to the dual space which is named $w$-convergence in [2]. In [2], authors also showed that $w$-convergence is stronger than $\Delta$-convergence. Ahmadi Kakavandi in [1] presented an equivalent definition of $w$-convergence in complete $\mathrm{CAT}(0)$ spaces without using of dual space, as follows:

Definition 2.2. [1] A sequence $\left(x_{n}\right)$ in the complete $\mathrm{CAT}(0)$ space $(X, d)$ $w$-converges to $x \in X \quad$ if $\limsup _{n \rightarrow \infty}\left\langle\overrightarrow{x x_{n}}, \overrightarrow{x y}\right\rangle=0, \quad \forall y \in X$.

$w$-convergence is equivalent to the weak convergence in Hilbert space $H$, because if $(.,$.$) is the inner product in Hilbert space H$, then

$$
2\langle\overrightarrow{x z}, \overrightarrow{x y}\rangle=d^{2}(x, y)+d^{2}(z, x)-d^{2}(z, y)=2(x-z, x-y) .
$$

We must notice that any bounded sequence does not have a subsequence that is $w$-convergent. It is obvious that convergence in the metric implies $w$-convergence, and in [2] it has been shown that $w$-convergence implies $\Delta$ convergence but the converse is not valid (see [1]). However Ahmadi Kakavandi [1] proved the following characterization of $\Delta$-convergence. 
Lemma 2.3. [1] $A$ bounded sequence $\left(x_{n}\right)$ in Hadamard space $(X, d), \Delta$ converges to $x \in X$ if and only if $\lim _{\sup _{n \rightarrow \infty}}\left\langle\overrightarrow{x x_{n}}, \overrightarrow{x y}\right\rangle \leq 0, \quad \forall y \in X$.

In the sequel, we denote $w$-convergence by $\rightarrow$ and strong convergence by $\rightarrow$.

\section{Maximal Monotone Operators}

Let $X$ be a complete CAT(0) space with dual $X^{*}$ and $A: X \rightarrow 2^{X^{*}}$ be a multi-valued operator with domain $\mathbb{D}(A):=\{x \in X: A x \neq \emptyset\}$, range $\mathbb{R}(A):=\bigcup_{x \in X} A x, \quad A^{-1}\left(x^{*}\right):=\left\{x \in X: x^{*} \in A x\right\}$ and graph $\operatorname{gra}(A):=\left\{\left(x, x^{*}\right) \in X \times X^{*}: x \in \mathbb{D}(A), x^{*} \in A x\right\}$.

Definition 3.1. Let $X$ be a Hadamard space with dual space $X^{*}$. The multivalued operator $A: X \rightarrow 2^{X^{*}}$ is monotone if and only if

$$
\left\langle x^{*}-y^{*}, \overrightarrow{y x}\right\rangle \geq 0
$$

for all $x, y \in \mathbb{D}(A), x^{*} \in A x, y^{*} \in A y$.

Definition 3.2. Let $X$ be a Hadamard space with dual $X^{*}$. The multi-valued monotone operator $A: X \rightarrow 2^{X^{*}}$ is maximal if there exists no monotone operator $B: X \rightarrow 2^{X^{*}}$ such that $\operatorname{gra}(B)$ properly contains $\operatorname{gra}(A)$, i.e. for any $\left(y, y^{*}\right) \in X \times X^{*}$, the inequality $\left\langle x^{*}-y^{*}, \overrightarrow{y x}\right\rangle \geq 0$, for all $\left(x, x^{*}\right) \in \operatorname{gra}(A)$ implies $y^{*} \in A y$.

In this section, we show that the graph of a maximal monotone operator is sequentialy weakly-strongly closed in $X \times X^{*}$, i.e. if $\left(x_{n}, x_{n}^{*}\right) \in \operatorname{gra}(A) \forall n \in \mathbb{N}$, $x_{n} \rightarrow x$ and $\left(x_{n}^{*}\right) \subset X^{*}$ converges to $x^{*} \in X^{*}$ in metric $D$ then $x^{*} \in A x$.

Lemma 3.3. Let $X$ be a Hadamard space with dual $X^{*}$ then

$$
\left|\left\langle x^{*}-y^{*}, \overrightarrow{y x}\right\rangle\right| \leq D\left(x^{*}, y^{*}\right) d(x, y), \quad \text { for all } x, y \in X, x^{*}, y^{*} \in X^{*} .
$$

Proof. let $x, y \in X, x^{*}, y^{*} \in X^{*}$. Consider $t, s \in \mathbb{R}$ and $a, b, c, d \in X$ such 
that $x^{*}=[t \overrightarrow{a b}]$ and $y^{*}=[\overrightarrow{s c d}]$. If $x=y$ that is clear, suppose $x \neq y$, then

$$
\begin{aligned}
&\left|\left\langle x^{*}-y^{*}, \overrightarrow{y x}\right\rangle\right|=|\langle[t \overrightarrow{a b}]-[s \overrightarrow{c d}], \overrightarrow{y x}\rangle| \\
&=\mid\langle t\langle\overrightarrow{a b}, \overrightarrow{y \vec{x}}\rangle-s\langle\overrightarrow{c d}, \overrightarrow{y \vec{x}}\rangle| \\
&=\mid\langle t\langle\overrightarrow{a b}, \overrightarrow{y a}\rangle+t\langle\overrightarrow{a b}, \overrightarrow{a x}\rangle-s\langle\overrightarrow{c d}, \overrightarrow{y c}\rangle-s\langle\overrightarrow{c d}, \overrightarrow{c x}\rangle| \\
&=|t\langle\overrightarrow{a b}, \overrightarrow{a x}\rangle-t\langle\overrightarrow{a b}, \overrightarrow{a y}\rangle-s\langle\overrightarrow{c d}, \overrightarrow{c x}\rangle+s\langle\overrightarrow{c d}, \overrightarrow{c y}\rangle| \\
&=d(x, y)\left|\frac{(\Theta(t, a, b)(x)-\Theta(s, c, d)(x))-(\Theta(t, a, b)(y)-\Theta(s, c, d)(y))}{d(x, y)}\right| \\
& \leq d(x, y) \sup \left\{\begin{aligned}
(\Theta(t, a, b)-\Theta(s, c, d))(u)-(\Theta(t, a, b)-\Theta(s, c, d))(v) \\
\leq
\end{aligned}\right. \\
&=d(x, y) L(\Theta(t, a, b)-\Theta(s, c, d)) \\
&=d(x, y) D([t \overrightarrow{a b}],[s \overrightarrow{c d}]) \\
&=d(x, y) D\left(x^{*}, y^{*}\right) .
\end{aligned}
$$

Theorem 3.4. Let $X$ be a Hadamard space with dual $X^{*}$ and $A: X \rightarrow 2^{X^{*}}$ be a multi-valued maximal monotone operator. Suppose $\left(x_{n}, x_{n}^{*}\right) \in \operatorname{gra}(A)$ for all $n \in \mathbb{N}$ such that $\left(x_{n}\right)$ is a bounded sequence in $X$ that is $w$-convergent to $x \in X$ and $\left(x_{n}^{*}\right) \subset X^{*}$ converges to $x^{*} \in X^{*}$ in metric $D$ then $x^{*} \in A x$.

Proof. By Lemma 3.3, for all $n \in \mathbb{N}$ and all $\left(y, y^{*}\right) \in \operatorname{gra}(A)$, we have

$$
\begin{aligned}
\left|\left\langle x_{n}^{*}-y^{*}, \overrightarrow{y x_{n}}\right\rangle-\left\langle x^{*}-y^{*}, \overrightarrow{y x}\right\rangle\right| & \\
& =\left|\left\langle x_{n}^{*}-x^{*}, \overrightarrow{y x_{n}}\right\rangle+\left\langle x^{*}-y^{*}, \overrightarrow{y x_{n}}\right\rangle+\left\langle x^{*}-y^{*}, \overrightarrow{x y}\right\rangle\right| \\
& =\left|\left\langle x_{n}^{*}-x^{*}, \overrightarrow{y x_{n}}\right\rangle+\left\langle x^{*}-y^{*}, \overrightarrow{x x_{n}}\right\rangle\right| \\
& \leq\left|\left\langle x_{n}^{*}-x^{*}, \overrightarrow{y x_{n}}\right\rangle\right|+\left|\left\langle x^{*}-y^{*}, \overrightarrow{x x_{n}}\right\rangle\right| \\
& \leq D\left(x_{n}^{*}, x^{*}\right) d\left(x_{n}, y\right)+\left|\left\langle x^{*}-y^{*}, \overrightarrow{x x_{n}}\right\rangle\right| .
\end{aligned}
$$

Let $n \rightarrow \infty$, we get

$$
\left\langle x_{n}^{*}-y^{*}, \overrightarrow{y x_{n}}\right\rangle \rightarrow\left\langle x^{*}-y^{*}, \overrightarrow{y x}\right\rangle
$$

On the other hand, by monotonicity of $A$, for all $\left(y, y^{*}\right) \in \operatorname{gra}(A)$, we have

$$
0 \leq\left\langle x_{n}^{*}-y^{*}, \overrightarrow{y x_{n}}\right\rangle, \quad \forall n \in \mathbb{N},
$$

which, by (3.1), implies

$$
0 \leq\left\langle x^{*}-y^{*}, \overrightarrow{y x}\right\rangle, \quad \forall\left(y, y^{*}\right) \in \operatorname{gra}(A) .
$$


Hence, the maximality of $A$ implies $x^{*} \in A x$.

We say that a subset $C$ of Hadamard space $X$ is $w$-sequentially closed if for any sequence $\left(x_{n}\right) \subset C$ that $x_{n} \rightarrow x$, we have $x \in C$. It is clear that every $w$-sequentially closed subset of $X$ is closed. By Theorem 3.4, it is easy to verify that if $A: X \rightarrow 2^{X^{*}}$ be a multi-valued maximal monotone operator then $A^{-1}\left(x^{*}\right)$ is a $w$-sequentially closed subset of Hadamard space $X$, for any $x^{*} \in X^{*}$.

\section{Halpern-type Proximal Point Algorithm}

One of the most important problems in monotone operator theory is finding a zero of a maximal monotone operator. This problem can be formulated in Hadamard space as follows:

$$
\text { Find } x \in X \text {, such that } \mathbf{0} \in A(x) \text {, }
$$

where $A: X \rightarrow 2^{X^{*}}$ is a monotone operator on the Hadamard space $X$ and $\mathbf{0}$ is the zero of dual space $X^{*}$.

Let $X$ be a Hadamard space with dual $X^{*}$ and $A: X \rightarrow 2^{X^{*}}$ be a multivalued operator. We say that $A$ satisfies the range condition if for every $y \in X$ and every $\alpha>0$, there exists a point $x \in X$ such that $[\alpha \overrightarrow{x y}] \in A x$. It is known that if $A$ is a maximal monotone operator on the Hilbert space $H$ then $\mathbb{R}(I+\lambda A)=H, \quad \forall \lambda>0$, where $I$ is identity operator. Thus, every maximal monotone operator $A$ on a Hilbert space satisfies the range condition.

Lemma 4.1. If $A$ is a monotone operator on a Hadamard space $X$ that satisfies the range condition then for every $y \in X$ and every $\alpha>0$, there exists a unique point $x \in X$ such that $[\alpha \overrightarrow{x y}] \in A x$.

Proof. If there exists $x, z \in X$ such that $[\alpha \overrightarrow{x y}] \in A x$ and $[\alpha \overrightarrow{z y}] \in A z$, then by monotonicity of $A$, we have

$$
\begin{aligned}
0 & \leq 2\langle[\alpha \vec{x} \vec{y}]-[\alpha \overrightarrow{z y}], \overrightarrow{z x}\rangle \\
& =2 \alpha\langle\overrightarrow{x y}, \overrightarrow{z x}\rangle-2 \alpha\langle\overrightarrow{z y}, \overrightarrow{z x}\rangle \\
& =\alpha\left(d^{2}(y, z)-d^{2}(x, z)-d^{2}(y, x)\right)-\alpha\left(d^{2}(x, z)+d^{2}(y, z)-d^{2}(y, x)\right) \\
& =-2 d^{2}(x, z)
\end{aligned}
$$

which implies $x=z$.

We do not know if every maximal monotone operator $A: X \rightarrow 2^{X^{*}}$ satisfies the range condition when $X$ is a Hadamard space. 
Let $A: X \rightarrow 2^{X^{*}}$ be a multi-valued maximal monotone operator on the Hadamard space $X$ with dual $X^{*}$ that satisfies the range condition, $\left(\lambda_{n}\right)$ is a sequence of positive real numbers, $\left(\alpha_{n}\right)$ is a sequence in $] 0,1[$ and $u \in X$. Halpern-type proximal point algorithm for maximal monotone operator $A$ in Hadamard space $X$ is the sequence generated by

$$
\left\{\begin{array}{l}
{\left[\frac{1}{\lambda_{n}} \overrightarrow{x_{n+1}\left(\alpha_{n} u \oplus\left(1-\alpha_{n}\right) x_{n}\right)}\right] \in A x_{n+1}} \\
x_{0} \in X
\end{array}\right.
$$

Note that, since the operator $A$ satisfies the rang condition, the Halpern-type proximal point algorithm (4.2) is well-defined and also (4.2) is accordance with the Halpern-type proximal point algorithm

$\left\{\begin{array}{l}x_{n+1} \in\left(I+\lambda_{n} A\right)^{-1}\left(\alpha_{n} u+\left(1-\alpha_{n}\right) x_{n}\right), \quad \text { where } I \text { is the identity operator } \\ x_{0} \in X .\end{array}\right.$ in a Hilbert space that is considered by $[5,6,7,8,9,25,33,35]$.

The aim of this section is to prove strong convergence of the sequence generated by the Halpern-type proximal point algorithm (4.2) to an element of $A^{-1}(\mathbf{0})$, where $\mathbf{0}$ is the zero of dual space. To this purpose, we need to the following lemmas.

Lemma 4.2. Let $X$ be a $C A T(0)$ space, $x, y \in X$ and $t \in] 0,1[$. Then,

$$
\langle\overrightarrow{y z}, \overrightarrow{(t x \oplus(1-t) y) y}\rangle \leq t\langle\overrightarrow{y z}, \overrightarrow{x y}\rangle, \quad(z \in X) .
$$

Proof. Let $z \in X$. By Lemma 2.1 we have,

$$
\begin{aligned}
2(\langle\overrightarrow{y z}, \overrightarrow{(t x \oplus(1-t) y) y}\rangle-t\langle\overrightarrow{y z}, \overrightarrow{x y}\rangle)= & \left(d^{2}((t x \oplus(1-t) y), z)-d^{2}((t x \oplus(1-t) y), y)\right. \\
& \left.-d^{2}(y, z)\right)-t\left(d^{2}(x, z)-d^{2}(x, y)-d^{2}(y, z)\right) \\
\leq & t d^{2}(x, z)+(1-t) d^{2}(y, z)-t(1-t) d^{2}(x, y) \\
& -t^{2} d^{2}(x, y)-d^{2}(y, z)-t d^{2}(x, z)+t d^{2}(x, y) \\
& +t d^{2}(y, z) \\
= & 0,
\end{aligned}
$$

which implies the desired inequality.

Lemma 4.3. Let $X$ be a $C A T(0)$ space and $\left(x_{n}\right)$ be a bounded sequence in $X$ that $\Delta$-converges to $X$. Then,

$$
d^{2}(x, y) \leq \liminf _{n} d^{2}\left(x_{n}, y\right) \quad(y \in X)
$$


Proof. By Lemma 2.3, for all $y \in X$, we get

$$
\begin{aligned}
d^{2}(x, y)-\liminf _{n} d^{2}\left(x_{n}, y\right) & =\limsup _{n}\left(d^{2}(x, y)-d^{2}\left(x_{n}, y\right)\right) \\
& \leq \limsup _{n}\left(d^{2}\left(x_{n}, x\right)+d^{2}(x, y)-d^{2}\left(x_{n}, y\right)\right) \\
& =\limsup _{n}\left\langle\overrightarrow{x x_{n}}, \overrightarrow{x y}\right\rangle \leq 0,
\end{aligned}
$$

that is the desired inequality.

Let $X$ be a Hadamard space with dual $X^{*}, A: X \rightarrow 2^{X^{*}}$ be a multivalued monotone operator such that satisfies the range condition and $C>0$ and $u \in X$ are fixed. Then by Lemma 4.1, for each $t \in] 0,1[$ and each $x \in X$, there exists a unique point $z_{t, x}$ such that $\left[\frac{1}{C} z_{t, x}(t u \oplus(1-t) x)\right] \in A\left(z_{t, x}\right)$. Thus, for every $t \in] 0,1\left[\right.$, we can define the mapping $S_{t}: X \rightarrow X$ with $S_{t}(x)=z_{t, x} \quad \forall x \in X$. In the following, we show that, for every $\left.t \in\right] 0,1\left[, S_{t}\right.$ has a unique fixed point $z_{t} \in X$.

Proposition 4.4. For each $t \in] 0,1\left[\right.$, $S_{t}$ has a unique fixed point $z_{t} \in X$.

Proof. Let $x, y \in X$, then

$\left[\frac{1}{C} \overrightarrow{S_{t}(x)(t u \oplus(1-t) x)}\right] \in A\left(S_{t}(x)\right)$ and $\left[\frac{1}{C} \overrightarrow{S_{t}(y)(t u \oplus(1-t) y)}\right] \in A\left(S_{t}(y)\right)$.

By monotonicity of $A$, we have

$$
\begin{aligned}
2\left\langle\overrightarrow{S_{t}(x)(t u \oplus(1-t) x)}, \overrightarrow{S_{t}(x) S_{t}(y)}\right\rangle & \leq 2\left\langle\overrightarrow{S_{t}(y)(t u \oplus(1-t) y)}, \overrightarrow{S_{t}(x) S_{t}(y)}\right\rangle \\
& =2\left\langle\overrightarrow{S_{t}(y)(t u \oplus(1-t) x)}, \overrightarrow{S_{t}(x) S_{t}(y)}\right\rangle \\
+ & 2\left\langle\overrightarrow{(t u \oplus(1-t) x)(t u \oplus(1-t) y)}, \overrightarrow{S_{t}(x) S_{t}(y)}\right\rangle
\end{aligned}
$$

which implies,

$$
\begin{aligned}
2 d^{2}\left(S_{t}(x), S_{t}(y)\right) & \leq 2\left\langle\overrightarrow{(t u \oplus(1-t) x)(t u \oplus(1-t) y)}, \overrightarrow{S_{t}(x) S_{t}(y)}\right\rangle \\
& \leq 2 d(t u \oplus(1-t) x, t u \oplus(1-t) y) d\left(S_{t}(x) S_{t}(y)\right) .
\end{aligned}
$$

Hence,

$$
d\left(S_{t}(x), S_{t}(y)\right) \leq d(t u \oplus(1-t) x, t u \oplus(1-t) y) \leq(1-t) d(x, y)
$$

Thus, for each $t \in] 0,1\left[, S_{t}\right.$ is a contraction. Consequently, by Banach's Contraction Principle, for each $t \in] 0,1\left[, S_{t}\right.$ has a unique fixed point that is named $z_{t}$ 
In the following, we show that $\left(z_{t}\right)$ converges strongly to $P_{A^{-1}(\mathbf{0})} u$, where $P_{A^{-1}(\mathbf{0})}$ is the metric projection on $A^{-1}(\mathbf{0})$.

Theorem 4.5. [15] Let $C$ be a nonempty convex subset of a CAT(0) space $X, x \in X$ and $u \in C$. Then $u=P_{C} x$ if and only if

$$
\langle\overrightarrow{x u}, \overrightarrow{u y}\rangle \geq 0, \quad \forall y \in C .
$$

We say that a Hadamard space $X$ satisfies the condition $Q$ if every bounded sequence in $X$ has a subsequence that is $w$-convergent.

It is said that a Hadamard space $(X, d)$ satisfies the $(S)$ property if for any $(x, y) \in X \times X$ there exists a point $y_{x} \in X$ such that $[\overrightarrow{x y}]=\left[\overrightarrow{y_{x} \vec{x}}\right]$. Hilbert spaces and symmetric Hadamard manifold satisfy the (S) property (see [1], Definition 2.7). Lemma 2.8 of [1] implies that if a Hadamard space $(X, d)$ satisfies the $(S)$ property then it satisfies the condition $Q$ because every bounded sequence in a Hadamard space $(X, d)$ has a $\Delta$-convergent subsequence. Also, the proper Hadamard spaces satisfy the condition $Q$ (see [1], Propositions 4.3 and 4.4).

The following theorem is a version of Browder convergence theorem (see [11]) for Halpern-type proximal point algorithm.

Theorem 4.6. Let $X$ be a Hadamard space with dual $X^{*}$ that satisfies the condition $Q$ and $A: X \rightarrow 2^{X^{*}}$ be a multi-valued maximal monotone operator that satisfies the range condition and $A^{-1}(\mathbf{0}) \neq \emptyset$ is convex. Then $\left(z_{t}\right)$ converges strongly to $P_{A^{-1}(\mathbf{0})} u$ as $t \rightarrow 0$.

Proof. For each $t \in] 0,1\left[,\left[\frac{1}{C} \overrightarrow{z_{t}\left(t u \oplus(1-t) z_{t}\right)}\right] \in A\left(z_{t}\right)\right.$. By monotonicity of $A$, for all $q \in A^{-1}(\mathbf{0})$, we have

$$
\begin{aligned}
0 & \leq 2\left\langle\overrightarrow{z_{t}\left(t u \oplus(1-t) z_{t}\right.}, \overrightarrow{q z_{t}}\right\rangle \\
& =d^{2}\left(t u \oplus(1-t) z_{t}, q\right)-d^{2}\left(z_{t}, q\right)-d^{2}\left(t u \oplus(1-t) z_{t}, z_{t}\right),
\end{aligned}
$$

hence,

$$
\begin{aligned}
d^{2}\left(z_{t}, q\right) & \leq d^{2}\left(t u \oplus(1-t) z_{t}, q\right) \\
& \leq t d^{2}(u, q)+(1-t) d^{2}\left(z_{t}, q\right),
\end{aligned}
$$

which implies,

$$
d^{2}\left(z_{t}, q\right) \leq d^{2}(u, q), \quad \forall q \in A^{-1}(\mathbf{0}) .
$$

In particular, $\left\{z_{t}\right\}$ is bounded. Thus, by the condition $Q$, there exists a subsequence of $\left(z_{t}\right)$ that is $w$-convergent. Moreover,

$\lim _{t \rightarrow 0} D\left(\left[\frac{1}{C} \overrightarrow{z_{t}\left(t u \oplus(1-t) z_{t}\right)}\right], \mathbf{0}\right)=\lim _{t \rightarrow 0} \frac{1}{C} d\left(t u \oplus(1-t) z_{t}, z_{t}\right)=\lim _{t \rightarrow 0} \frac{t}{C} d\left(u, z_{t}\right)=0$. 
Now, if $\left(t_{n}\right)$ is a sequence in ] $0,1\left[\right.$ such that $t_{n} \rightarrow 0$ and $\left(z_{t_{n}}\right)$ is $w$-convergent to $z$. Then, by (4.4) and Theorem 3.4, we get $z \in A^{-1}(\mathbf{0})$. Thus, by (4.4) and Lemma 4.3 , we obtain

$$
d^{2}(z, q) \leq \liminf _{n} d^{2}\left(z_{t_{n}}, q\right) \leq d^{2}(u, q), \quad \forall q \in A^{-1}(\mathbf{0}),
$$

which, for all $q \in A^{-1}(\mathbf{0})$, implies,

$$
\begin{aligned}
\langle\overrightarrow{z u}, \overrightarrow{z q}\rangle & =\langle\overrightarrow{z u}, \overrightarrow{z u}\rangle+\langle\overrightarrow{z u}, \overrightarrow{u q}\rangle \\
& =d^{2}(z, q)-d^{2}(u, q) \\
& \leq 0 .
\end{aligned}
$$

Hence, by Theorem 4.5, $z=P_{A^{-1}(\mathbf{0})} u$. The arbitrariness of the subsequence $\left(z_{t_{n}}\right)$ of $\left(z_{t}\right)$ ensures that $\left(z_{t}\right)$ indeed $w$-converges to $P_{A^{-1}(\mathbf{0})} u$, as $t \rightarrow 0$. Now, we prove the strong convergence of $\left(z_{t}\right)$.

By monotonicity of $A$ and Lemma 4.2 , for all $q \in A^{-1}(\mathbf{0})$, we have

$$
\begin{aligned}
0 & \leq 2\left\langle\overrightarrow{z_{t}\left(t u \oplus(1-t) z_{t}\right)}, \overrightarrow{q z_{t}}\right\rangle \\
& \leq t\left\langle\overrightarrow{z_{t} u}, \overrightarrow{q z_{t}}\right\rangle \\
& =t\left\langle\overrightarrow{z_{t}}, \overrightarrow{q z_{t}}\right\rangle+t\left\langle\overrightarrow{q u}, \overrightarrow{q z_{t}}\right\rangle,
\end{aligned}
$$

which implies

$$
d^{2}\left(z_{t}, q\right) \leq\left\langle\overrightarrow{q u}, \overrightarrow{q z_{t}}\right\rangle \quad \forall q \in A^{-1}(\mathbf{0})
$$

In particular,

$$
d^{2}\left(z_{t}, P_{A^{-1}(\mathbf{0})} u\right) \leq\left\langle\overrightarrow{\left(P_{A^{-1}(\mathbf{0})} u\right) u}, \overrightarrow{\left(P_{A^{-1}(\mathbf{0})} u\right) z_{t}}\right\rangle .
$$

Letting $t \rightarrow 0$, we get $d^{2}\left(z_{t}, P_{A^{-1}(\mathbf{0})} u\right) \rightarrow 0$, that is the desired result.

Lemma 4.7. [15] Let $(X, d)$ be a CAT(0) space and $a, b, c \in X$. Then for each $\lambda \in[0,1]$,

$$
d^{2}(\lambda a \oplus(1-\lambda) b, c) \leq \lambda^{2} d^{2}(a, c)+(1-\lambda)^{2} d^{2}(b, c)+2 \lambda(1-\lambda)\langle\overrightarrow{a c}, \overrightarrow{b c}\rangle .
$$

Theorem 4.8. Let $X$ be a Hadamard space with dual $X^{*}$ that satisfies the condition $Q$ and $A: X \rightarrow 2^{X^{*}}$ be a multi-valued maximal monotone operator such that satisfies the range condition and $A^{-1}(\mathbf{0}) \neq \emptyset$ is convex. If $\left(\left(x_{n}, x_{n}^{*}\right)\right)$ is a sequence in graph of $A$ such that $\left(x_{n}\right)$ is bounded and $\lim _{n} D\left(x_{n}^{*}, \mathbf{0}\right)=0$, then

$$
\limsup _{n}\left\langle\overrightarrow{u p}, \overrightarrow{x_{n} p}\right\rangle \leq 0, \text { where } p=P_{A^{-1}(\mathbf{0})} u \text {. }
$$


Proof. For each $t \in] 0,1\left[\right.$, there exists a unique point $z_{t} \in X$ such that $\left[\frac{1}{C} \overrightarrow{z_{t}\left(t u \oplus(1-t) z_{t}\right)}\right] \in A\left(z_{t}\right)$. By Theorem 4.6, as $t \rightarrow 0,\left(z_{t}\right)$ converges strongly to $p=P_{A^{-1}(\mathbf{0})} u$. By monotonicity of $A$, for each $t \in(0,1)$ and all $n \in \mathbb{N}$, we have

$$
0 \leq \frac{2}{C}\left\langle\overrightarrow{z_{t}\left(t u \oplus(1-t) z_{t}\right)}-x_{n}^{*}, \overrightarrow{x_{n} z_{t}}\right\rangle .
$$

which, by Lemma 4.7, implies

$$
\begin{aligned}
d^{2}\left(z_{t}, x_{n}\right)+2\left\langle x_{n}^{*}, \overrightarrow{x_{n} z_{t}}\right\rangle \leq & d^{2}\left(t u \oplus(1-t) z_{t}, x_{n}\right)-t^{2} d^{2}\left(u, z_{t}\right) \\
\leq & t^{2} d^{2}\left(u, x_{n}\right)+(1-t)^{2} d^{2}\left(z_{t}, x_{n}\right)+2 t(1-t)\left\langle\overrightarrow{u x_{n}}, \overrightarrow{z_{t} x_{n}}\right\rangle \\
= & t^{2} d^{2}\left(u, x_{n}\right)+(1-t)^{2} d^{2}\left(z_{t}, x_{n}\right)+2 t(1-t)\left\langle\overrightarrow{u z_{t}}, \overrightarrow{z_{t} x_{n}}\right\rangle \\
& +2 t(1-t) d^{2}\left(z_{t}, x_{n}\right) \\
= & t^{2} d^{2}\left(u, x_{n}\right)+\left(1-t^{2}\right) d^{2}\left(z_{t}, x_{n}\right)+2 t(1-t)\left\langle\overrightarrow{u z_{t}}, \overrightarrow{z_{t} x_{n}}\right\rangle .
\end{aligned}
$$

Thus, for each $t \in(0,1)$ and all $n \in \mathbb{N}$, we get

$$
\begin{aligned}
2 t(1-t)\left\langle\overrightarrow{u z_{t}}, \overrightarrow{x_{n} z_{t}}\right\rangle & \leq t^{2} d^{2}\left(u, x_{n}\right)+2\left\langle x_{n}^{*}, \overrightarrow{z_{t} x_{n}}\right\rangle \\
& \leq t^{2} d^{2}\left(u, x_{n}\right)+2 D\left(x_{n}^{*}, \mathbf{0}\right) d\left(z_{t}, x_{n}\right) .
\end{aligned}
$$

Hence, for each $t \in(0,1)$, we obtain

$$
\limsup _{n}\left\langle\overrightarrow{u z_{t}}, \overrightarrow{x_{n} z_{t}}\right\rangle \leq \frac{t}{2(1-t)} \limsup _{n} d^{2}\left(u, x_{n}\right)
$$

On the other hand, by the continuity of $d$,

$$
\left\langle\overrightarrow{u z_{t}}, \overrightarrow{x_{n} z_{t}}\right\rangle \rightarrow\left\langle\overrightarrow{u p}, \overrightarrow{x_{n} p}\right\rangle \text { as } t \rightarrow 0, \quad \text { uniformly respect to } n \text {. }
$$

Therefore, for any number $\varepsilon>0$, there exists $\delta>0$ such that

$$
\left\langle\overrightarrow{u p}, \overrightarrow{x_{n} p}\right\rangle \leq \varepsilon+\left\langle\overrightarrow{u z_{t}}, \overrightarrow{x_{n} z_{t}}\right\rangle,
$$

for all $0<t<\delta$ and all $n \in \mathbb{N}$. This implies that

$$
\underset{n}{\limsup }\left\langle\overrightarrow{u p}, \overrightarrow{x_{n} p}\right\rangle \leq \varepsilon+\underset{n}{\limsup }\left\langle\overrightarrow{u z_{t}}, \overrightarrow{x_{n} z_{t}}\right\rangle \leq \varepsilon+\frac{t}{2(1-t)} \limsup _{n} d^{2}\left(u, x_{n}\right) .
$$

Letting $t \rightarrow 0$, we get

$$
\limsup _{n}\left\langle\overrightarrow{u p}, \overrightarrow{x_{n} p}\right\rangle \leq \varepsilon .
$$

Hence, as $\varepsilon \rightarrow 0$, we deduce $\lim \sup _{n}\left\langle\overrightarrow{u p}, \overrightarrow{x_{n} p}\right\rangle \leq 0$. 
Lemma 4.9. [34] Let $\left(s_{n}\right)$ be a sequence of nonnegative real numbers, $\left(\alpha_{n}\right)$ a sequence of real numbers in $[0,1]$ with $\sum_{n=1}^{\infty} \alpha_{n}=\infty,\left(u_{n}\right)$ a sequence of nonnegative real numbers with $\sum_{n=1}^{\infty} u_{n}<\infty$, and $\left(t_{n}\right)$ a sequence of real numbers with $\lim \sup _{n} t_{n} \leq 0$. Suppose that

$$
s_{n+1} \leq\left(1-\alpha_{n}\right) s_{n}+\alpha_{n} t_{n}+u_{n}
$$

for all $n \in \mathbb{N}$. Then $\lim _{n \rightarrow \infty} s_{n}=0$.

The following lemma is a direct application of the well-known Stolz-Cesàro theorem.

Lemma 4.10. Suppose that $\left(a_{n}\right)$ and $\left(b_{n}\right)$ are positive sequences such that $\sum_{n=1}^{+\infty} b_{n}=+\infty$ and $\lim _{n \rightarrow+\infty} \frac{a_{n}}{b_{n}}=0$, then $\lim _{m \rightarrow+\infty} \frac{\sum_{n=1}^{m} a_{n}}{\sum_{n=1}^{m} b_{n}}=0$.

Theorem 4.11. Let $X$ be a Hadamard space with dual $X^{*}$ that satisfies the condition $Q$ and $A: X \rightarrow 2^{X^{*}}$ be a multi-valued maximal monotone operator that satisfies the range condition and $A^{-1}(\mathbf{0}) \neq \emptyset$ is convex. If $\left(x_{n}\right)$ generated by (4.2) such that

$$
\begin{aligned}
D^{2}\left(\left[\frac{1}{\lambda_{n}} \overrightarrow{x_{n+1}\left(\alpha_{n} u \oplus\left(1-\alpha_{n}\right) x_{n}\right)}\right], \mathbf{0}\right) \leq & D^{2}\left(\left[\frac{1}{\lambda_{n-1}} \overrightarrow{x_{n}\left(\alpha_{n-1} u \oplus\left(1-\alpha_{n-1}\right) x_{n-1}\right)}\right], \mathbf{0}\right) \\
& +\theta_{n},
\end{aligned}
$$

where $\left(\theta_{n}\right)$ is a positive sequence with $\sum_{n=1}^{\infty} \theta_{n}<\infty, \sum_{n=1}^{\infty} \lambda_{n}^{2}=\infty$ and $\frac{\alpha_{n}}{\lambda_{n}^{2}} \rightarrow 0$, then

(1) $D\left(\left[\frac{1}{\lambda_{n}} \overrightarrow{x_{n+1}\left(\alpha_{n} u \oplus\left(1-\alpha_{n}\right) x_{n}\right)}\right], \mathbf{0}\right) \rightarrow 0$, as $n \rightarrow \infty$. Also, if $\left(x_{n_{k}}\right)$ is a subsequence of $\left(x_{n}\right)$, w-converges to $x$, then $x \in A^{-1}(\mathbf{0})$.

(2) If $\alpha_{n} \rightarrow 0$ and $\sum_{n=1}^{+\infty} \alpha_{n}=+\infty$, then $\left(x_{n}\right)$ converges strongly to $p=P_{A^{-1}(\mathbf{0})} u$.

Proof. Let $q \in A^{-1}(\mathbf{0})$. By monotonicity of $A$ and (4.2), we have

$$
0 \leq\left\langle\left[\frac{1}{\lambda_{n}} \overrightarrow{x_{n+1}\left(\alpha_{n} u \oplus\left(1-\alpha_{n}\right) x_{n}\right)}\right], \overrightarrow{q x_{n+1}}\right\rangle,
$$

which implies

$$
\left.\left.d^{2}\left(x_{n+1}, q\right)+d^{2}\left(x_{n+1}, \alpha_{n} u \oplus\left(1-\alpha_{n}\right) x_{n}\right)\right) \leq d^{2}\left(\alpha_{n} u \oplus\left(1-\alpha_{n}\right) x_{n}\right), q\right) .
$$

Thus

$$
\begin{aligned}
d^{2}\left(x_{n+1}, q\right) & \leq d^{2}\left(\alpha_{n} u \oplus\left(1-\alpha_{n}\right) x_{n}, q\right) \\
& \leq \alpha_{n} d^{2}(u, q)+\left(1-\alpha_{n}\right) d^{2}\left(x_{n}, q\right) \\
& \leq \max \left\{d^{2}(u, q), d^{2}\left(x_{1}, q\right)\right\} .
\end{aligned}
$$


So, $\left(x_{n}\right)$ is bounded. Let us to prove (1). By (4.6), we have

$$
\begin{aligned}
\left.d^{2}\left(x_{n+1}, \alpha_{n} u \oplus\left(1-\alpha_{n}\right) x_{n}\right)\right) & \left.\leq d^{2}\left(\alpha_{n} u \oplus\left(1-\alpha_{n}\right) x_{n}\right), q\right)-d^{2}\left(x_{n+1}, q\right) \\
& \leq \alpha_{n} d^{2}(u, q)+d^{2}\left(x_{n}, q\right)-d^{2}\left(x_{n+1}, q\right),
\end{aligned}
$$

that is

$\lambda_{n}^{2} D^{2}\left(\left[\frac{1}{\lambda_{n}} \overrightarrow{x_{n+1}\left(\alpha_{n} u \oplus\left(1-\alpha_{n}\right) x_{n}\right)}\right], \mathbf{0}\right) \leq \alpha_{n} d^{2}(u, q)+d^{2}\left(x_{n}, q\right)-d^{2}\left(x_{n+1}, q\right)$.

Moreover, by assumptions, for all $k>n$, we get

$$
\begin{aligned}
D^{2}\left(\left[\frac{1}{\lambda_{k}} \overrightarrow{x_{k+1}\left(\alpha_{k} u \oplus\left(1-\alpha_{k}\right) x_{k}\right)}\right], \mathbf{0}\right) \leq & D^{2}\left(\left[\frac{1}{\lambda_{n}} \overrightarrow{x_{n+1}\left(\alpha_{n} u \oplus\left(1-\alpha_{n}\right) x_{n}\right)}\right], \mathbf{0}\right) \\
& +\sum_{i=n+1}^{k} \theta_{i},
\end{aligned}
$$

which, by (4.7), implies

$$
\begin{aligned}
\lambda_{n}^{2} D^{2}\left(\left[\frac{1}{\lambda_{k}} \overrightarrow{x_{k+1}\left(\alpha_{k} u \oplus\left(1-\alpha_{k}\right) x_{k}\right)}\right], \mathbf{0}\right) \leq & \alpha_{n} d^{2}(u, q)+d^{2}\left(x_{n}, q\right)-d^{2}\left(x_{n+1}, q\right) \\
& +\lambda_{n}^{2} \sum_{i=n+1}^{k} \theta_{i} .
\end{aligned}
$$

Summing up from $n=1$ to $k$, after that dividing by $\sum_{n=1}^{k} \lambda_{n}^{2}$, then

$$
\begin{aligned}
D^{2}\left(\left[\frac{1}{\lambda_{k}} \overrightarrow{x_{k+1}\left(\alpha_{k} u \oplus\left(1-\alpha_{k}\right) x_{k}\right)}\right], \mathbf{0}\right) \leq & \frac{\sum_{n=1}^{k} \alpha_{n} d^{2}(u, q)}{\sum_{n=1}^{k} \lambda_{n}^{2}}+\frac{d^{2}\left(x_{1}, q\right)}{\sum_{n=1}^{k} \lambda_{n}^{2}} \\
& +\frac{\sum_{n=1}^{k} \lambda_{n}^{2} \sum_{i=n+1}^{\infty} \theta_{i}}{\sum_{n=1}^{k} \lambda_{n}^{2}} .
\end{aligned}
$$

Letting $k \rightarrow \infty$ then, by Lemma 4.10 , we get

$$
D\left(\left[\frac{1}{\lambda_{k}} \overrightarrow{x_{k+1}\left(\alpha_{k} u \oplus\left(1-\alpha_{k}\right) x_{k}\right)}\right], \mathbf{0}\right) \rightarrow 0 .
$$

Now, if $\left(x_{n_{k}}\right)$ is a subsequence of $\left(x_{n}\right)$ that $w$-converges to $x$, then by Theorem 3.4 , we get $x \in A^{-1}(\mathbf{0})$.

For prove (2), by Theorem 4.8 and part (1), we get

$$
\limsup _{n}\left\langle\overrightarrow{u p}, \overrightarrow{x_{n} p}\right\rangle \leq 0, \quad \text { where } \quad p=P_{A^{-1}(\mathbf{0})} u .
$$


On the other hand, by (4.6), and Lemma 4.7 we have

$$
\begin{aligned}
d^{2}\left(x_{n+1}, p\right) & \left.\leq d^{2}\left(\alpha_{n} u \oplus\left(1-\alpha_{n}\right) x_{n}\right), p\right) \\
& \leq \alpha_{n}^{2} d^{2}(u, p)+\left(1-\alpha_{n}\right)^{2} d^{2}\left(x_{n}, p\right)+2 \alpha_{n}\left(1-\alpha_{n}\right)\left\langle\overrightarrow{u p}, \overrightarrow{x_{n} p}\right\rangle \\
& \leq\left(1-\alpha_{n}\right) d^{2}\left(x_{n}, p\right)+\alpha_{n}\left(\alpha_{n} d^{2}(u, p)+\left(1-\alpha_{n}\right)\left\langle\overrightarrow{u p}, \overrightarrow{x_{n} p}\right\rangle\right),
\end{aligned}
$$

which, by the Lemma 4.9, the assumptions and (4.8), implies $d\left(x_{n+1}, p\right) \rightarrow 0$, that is the desired result.

Theorem 4.12. Let $X$ be a Hadamard space with dual $X^{*}$ that satisfies the condition $Q$ and $A: X \rightarrow 2^{X^{*}}$ be a multi-valued maximal monotone operator such that satisfies the range condition and $A^{-1}(\mathbf{0}) \neq \emptyset$ is convex. If $\left(x_{n}\right)$ generated by (4.2) such that

(i) $\left(\lambda_{n}\right)$ is a nondecreasing sequence such that $\sum_{n=1}^{\infty} \frac{1}{\lambda_{n} \lambda_{n-1}}<\infty$,

(ii) $\alpha_{n} \rightarrow 0$ and $\sum_{n=1}^{+\infty} \alpha_{n}=+\infty$,

then $\left(x_{n}\right)$ converges strongly to $p=P_{A^{-1}(\mathbf{0})} u$.

Proof. By a proof similar to Theorem 4.11, $\left(x_{n}\right)$ is bounded. By monotonicity of $A$ and (4.2), we have

$0 \leq \frac{1}{\lambda_{n}}\left\langle\left[\frac{1}{\lambda_{n-1}} \overrightarrow{x_{n}\left(\alpha_{n-1} u \oplus\left(1-\alpha_{n-1}\right) x_{n-1}\right)}\right]-\left[\frac{1}{\lambda_{n}} \overrightarrow{x_{n+1}\left(\alpha_{n} u \oplus\left(1-\alpha_{n}\right) x_{n}\right)}\right], \overrightarrow{x_{n+1} x_{n}}\right\rangle$

that is

$\frac{1}{\lambda_{n}^{2}}\left\langle\overrightarrow{x_{n+1}\left(\alpha_{n} u \oplus\left(1-\alpha_{n}\right) x_{n}\right)}, \overrightarrow{x_{n+1} x_{n}}\right\rangle \leq \frac{1}{\lambda_{n} \lambda_{n-1}}\left\langle\overrightarrow{x_{n}\left(\alpha_{n-1} u \oplus\left(1-\alpha_{n-1}\right) x_{n-1}\right)}, \overrightarrow{x_{n+1} x_{n}}\right\rangle$

which implies

$$
\begin{aligned}
& \frac{1}{\lambda_{n}^{2}} d^{2}\left(x_{n+1}, \alpha_{n} u \oplus\left(1-\alpha_{n}\right) x_{n}\right) \leq \frac{1}{\lambda_{n} \lambda_{n-1}} d^{2}\left(x_{n+1}, \alpha_{n-1} u \oplus\left(1-\alpha_{n-1}\right) x_{n-1}\right) \\
&+\frac{\alpha_{n}^{2}}{\lambda_{n}^{2}} d^{2}\left(u, x_{n}\right) \\
& \leq \frac{1}{\lambda_{n} \lambda_{n-1}} d^{2}\left(x_{n}, \alpha_{n-1} u \oplus\left(1-\alpha_{n-1}\right) x_{n-1}\right) \\
&+\frac{1}{\lambda_{n} \lambda_{n-1}} d^{2}\left(x_{n+1}, x_{n}\right)+\frac{\alpha_{n}^{2}}{\lambda_{n}^{2}} d^{2}\left(u, x_{n}\right) \\
&+\frac{2}{\lambda_{n} \lambda_{n-1}} d\left(x_{n+1}, x_{n}\right) d\left(x_{n}, \alpha_{n-1} u \oplus\left(1-\alpha_{n-1}\right) x_{n-1}\right) \\
& \leq \frac{1}{\lambda_{n-1}^{2}} d^{2}\left(x_{n}, \alpha_{n-1} u \oplus\left(1-\alpha_{n-1}\right) x_{n-1}\right) \\
&+M\left(\frac{1}{\lambda_{n} \lambda_{n-1}}+\frac{\alpha_{n}^{2}}{\lambda_{n}^{2}}\right),
\end{aligned}
$$


where

$M=\sup _{n}\left\{2 d\left(x_{n+1}, x_{n}\right) d\left(x_{n}, \alpha_{n-1} u \oplus\left(1-\alpha_{n-1}\right) x_{n-1}\right), d^{2}\left(x_{n+1}, x_{n}\right), d^{2}\left(u, x_{n}\right)\right\}$.

Hence

$$
\begin{aligned}
D^{2}\left(\left[\frac{1}{\lambda_{n}} \overrightarrow{x_{n+1}\left(\alpha_{n} u \oplus\left(1-\alpha_{n}\right) x_{n}\right)}\right], \mathbf{0}\right) \leq & D^{2}\left(\left[\frac{1}{\lambda_{n-1}} \overrightarrow{x_{n}\left(\alpha_{n-1} u \oplus\left(1-\alpha_{n-1}\right) x_{n-1}\right)}\right], \mathbf{0}\right) \\
& +\theta_{n},
\end{aligned}
$$

where $\theta_{n}=M\left(\frac{1}{\lambda_{n} \lambda_{n-1}}+\frac{\alpha_{n}^{2}}{\lambda_{n}^{2}}\right), \quad \forall n \in \mathbb{N}$. By the assumptions, we have $\sum_{n=1}^{\infty} \theta_{n}<\infty, \sum_{n=1}^{\infty} \lambda_{n}^{2}=\infty, \frac{\alpha_{n}}{\lambda_{n}^{2}} \rightarrow 0, \alpha_{n} \rightarrow 0$ and $\sum_{n=1}^{+\infty} \alpha_{n}=+\infty$. Hence, part (2) of Theorem 4.11 completes the proof.

\section{Acknowledgments:}

The authors are grateful to the referee for his(her) careful reading and valuable comments and suggestions. This work was supported by Higher Education Center of Eghlid.

\section{References}

[1] B. Ahmadi Kakavandi, Weak topologies in complete CAT(0) metric spaces, Proc. Amer. Math. Soc. 141 (2013), 1029-1039.

[2] B. Ahmadi Kakavandi, M. Amini, Duality and Subdifferential for Convex Functions on Complete CAT(0) Metric Spaces, Nonlinear Anal. 73 (2010) $3450-3455$.

[3] M. Bačák, The proximal point algorithm in metric spaces, Israel J. Math. 194 (2013), 689-701.

[4] I.D. Berg, I.G. Nikolaev, Quasilinearization and curvature of Alexandrov spaces, Geom. Dedicata 133 (2008) 195-218.

[5] O.A. Boikanyo, G. Morosanu, A proximal point algorithm converging strongly for general errors, Optim. Lett. 4 (2010) 635-641.

[6] O.A. Boikanyo, G. Morosanu, Modified Rockafellar's algorithms, Math. Sci. Res. J. 13 (2009) 101-122.

[7] O.A. Boikanyo, G. Morosanu, Inexact Halpern-type proximal point algorithm, J. Glob. Optim. 51 (2011) 11-26.

[8] O.A. Boikanyo, G. Morosanu, Four parameter proximal point algorithms, Nonlinear Anal. 74 (2011) 544-555. 
[9] O.A. Boikanyo, G. Morosanu, Strong convergence of a proximal point algorithm with bounded error sequence, Optim. Lett. 7 (2013) no. 2, 415420 .

[10] M. Bridson, A. Haefliger, Metric Spaces of Non-Positive Curvature. Fundamental Principles of Mathematical Sciences. Springer, Berlin 319 (1999).

[11] F.E. Browder, Convergence of approximants to fixed points of nonexpansive mappings in Banach spaces, Arch. Ration. Mech. Anal. 24 (1967) 82-90.

[12] H. Brézis, P.L. Lions, Produits infinis derésolvantes, Israel J. Math. 29 (1978) 329-345.

[13] K.S. Brown, Buildings. Springer, New York, (1989).

[14] D. Burago, Y. Burago, S. Ivanov, A Course in Metric Geometry, Graduate Studies in Math., 33, Amer. Math. Soc., Providence, RI (2001).

[15] H. Dehghan, J. Rooin, Metric projection and convergence theorems for nonexpansive mappings in Hadamard spaces, J. Nonlinear Convex Anal. (To appear)

[16] S. Dhompongsa, B. Panyanak, On $\Delta$-convergence theorems in CAT(0) spaces, Comput. Math. Appl. 56 (2008) 2572-2579.

[17] B. Djafari Rouhani, H. Khatibzadeh, On the proximal point algorithm, J. Optim. Theory Appl. 137 (2008) 411-417.

[18] R. Espínola, A. Fernández-León, CAT $(\kappa)$-spaces, weak convergence and fixed points, J.Math. Anal. Appl. 353 (2009) 410-427.

[19] K. Goebel, S. Reich, Uniform Convexity, Hyperbolic Geometry, and Nonexpansive Mappings. Monographs and Textbooks in Pure and Applied Mathematics, Marcel Dekker, Inc, New York, 83 (1984).

[20] M. Gromov, S.M. Bates, Metric structures for Riemannian and nonRiemannian spaces, with appendices by M. Katz, P. Pansu and S. Semmes, ed. by J. Lafontaine and P. Pansu, Progr. Math. 152, BirkhNauser, Boston (1999).

[21] O. Güler, On the convergence of the proximal point algorithm for convex minimization, SIAM J. Control Optim. 29 (1991) 403-419. 
[22] J. Jöst, Nonpositive curvature: Geometric and analytic aspects, Lectures Math. ETH ZNurich, BirkhNauser, Basel (1997).

[23] S. Kamimura, W. Takahashi, Approximating solutions of maximal monotone operators in Hilbert Spaces, J. Approx. Theory 106 (2000) 226-240.

[24] H. Khatibzadeh, Some remarks on the proximal point algorithm, J. Optim. Theory Appl. 153 (2012) 769-778.

[25] H. Khatibzadeh, S. Ranjbar, On the Strong Convergence of Halpern Type Proximal Point Algorithm, J. Optim. Theory Appl. 158 (2013) 385-396.

[26] H. Khatibzadeh and S. Ranjbar, Monotone operators and the proximal point algorithm in complete CAT(0) metric spaces, J. Aust. Math Soc. (Online published) doi: 10.1017/s1446788716000446.

[27] W.A. Kirk, Fixed point theorems in $\operatorname{CAT}(0)$ spaces and $\mathbb{R}$-trees, Fixed Point Theory Appl. 4 (2004) 309-316.

[28] W.A. Kirk, B. Panyanak, A concept of convergence in geodesic spaces, Nonlinear Anal. 68 (2008) 3689-3696.

[29] T.C. Lim, Remarks on some fixed point theorems, Proc. Amer. Math. Soc. 60 (1976) 179-182.

[30] B. Martinet, Régularisation d'Inéquations Variationnelles par Approximations Successives, Revue Franćaise d'Informatique et de Recherche Opérationnelle 3 (1970) 154-158.

[31] S. Ranjbar, W-convergence of the proximal point algorithm in complete CAT(0) metric spaces, Bull. Iranian Math. Soc. (To appear)

[32] R.T. Rockafellar, Monotone operators and the proximal point algorithm, SIAM J. Control Optim. 14 (1976) 877-898.

[33] F. Wang, H. Cui, On the contraction proximal point algorithms with multi parameters, J. Glob. Optim. 54 (2012) 485-491.

[34] H.K. Xu, Iterative algorithms for nonlinear operators, J. Lond. Math. Soc. 66 (2002) 240-256.

[35] H.K. Xu, A regularization method for the proximal point algorithm, J. Glob. Optim. 36 (2006) 115-125. 
Mohammad Taghi Heydari,

Department of Mathematics,

College of Sciences,

Yasouj University,

Yasouj 75914, Iran.

Email: heydari@yu.ac.ir,

and

Department of Mathematics,

College of Sciences,

Higher Education Center of Eghlid,

Eghlid, Iran.

Email: mt_heydari@yahoo.com

Sajad Ranjbar,

Department of Mathematics,

College of Sciences,

Higher Education Center of Eghlid,

Eghlid, Iran.

Email: sranjbar@eghlid.ac.ir, sranjbar74@yahoo.com.

Corresponding author. 